\title{
The potential of anti-malarial compounds derived from African medicinal plants, part III: an in silico evaluation of drug metabolism and pharmacokinetics profiling
}

Pascal Amoa Onguéné ${ }^{\dagger}$, Fidele Ntie-Kang ${ }^{2,3 \dagger}{ }^{+}$James Ajeck Mbah², Lydia Likowo Lifongo², Jean Claude Ndom', Wolfgang Sippl ${ }^{3}$ and Luc Meva'a Mbaze ${ }^{1 *}$

\begin{abstract}
Background: Malaria is an endemic disease affecting many countries in Tropical regions. In the search for compound hits for the design and/or development of new drugs against the disease, many research teams have resorted to African medicinal plants in order to identify lead compounds. Three-dimensional molecular models were generated for anti-malarial compounds of African origin (from 'weakly' active to 'highly' active), which were identified from literature sources. Selected computed molecular descriptors related to absorption, distribution, metabolism, excretion and toxicity (ADMET) of the phytochemicals have been analysed and compared with those of known drugs in order to access the 'drug-likeness' of these compounds.

Results: In the present study, more than 500 anti-malarial compounds identified from 131 distinct medicinal plant species belonging to 44 plant families from the African flora have been considered. On the basis of Lipinski's 'Rule of Five', about $70 \%$ of the compounds were predicted to be orally bioavailable, while on the basis of Jorgensen's 'Rule of Three', a corresponding $>80 \%$ were compliant. An overall drug-likeness parameter indicated that approximately $55 \%$ of the compounds could be potential leads for the development of drugs.
\end{abstract}

Conclusions: From the above analyses, it could be estimated that $>50 \%$ of the compounds exhibiting anti-plasmodial/ anti-malarial activities, derived from the African flora, could be starting points for drug discovery against malaria. The 3D models of the compounds have been included as an accompanying file and could be employed in virtual screening.

Keywords: Africa; Malaria; Medicinal plants; Metabolism; Natural products; Pharmacokinetics

\section{Background}

Malaria is an endemic disease which affects vast proportions of the populations of most Tropical countries (covering Africa, Asia and Latin America) [1,2]. The disease condition is caused by protozoans of the Plasmodium genus, mostly Plasmodium falciparum [3]. Statistics show that about half of the world's population is at risk of contracting malaria and that 1 to 2 million annual deaths (mostly amongst African children) can be attributed to

\footnotetext{
* Correspondence: Imbazze@yahoo.fr

${ }^{\dagger}$ Equal contributors

'Department of Chemistry, Faculty of Science, University of Douala, 00237 ,

P. O. Box 24157 Douala, Cameroon

Full list of author information is available at the end of the article
}

malaria alone [2-4]. In addition, the spread of the disease has been enhanced by the development of resistance in the anopheline vector against standard insecticides, amongst other factors, which have not unfortunately been put under check [5].

One promising way to fight malaria is to search for vaccines and new drugs, since no vaccine has yet been put in the market and the disease-causing parasites have developed resistant strains against existing chemotherapies [5-7]. It should however be mentioned that the process of discovering a drug is quite timely and costly [8]. One of the current approaches for shortening the time required and cutting down the cost for the discovery of lead 
compounds which potentially inhibit or modulate known drug targets is to incorporate computer-based methods like docking techniques, pharmacophore-based searches and neural networking [9-13]. Computer-based methods have also been incorporated in the prediction of likely metabolic pathways of drug molecules, as well as predict their pharmacokinetic profiles [14-17]. The absorption, distribution, metabolism, excretion and toxicity (ADMET) profile of a potential drug molecule should be known if it has to stand the chances of entering the market. Hence, assessing such information for lead compounds early enough would help eliminate molecules with predicted uninteresting profiles and eventually cut down the price of drug discovery [8].

With the accumulation of 'wet lab' biodata on drug metabolism and pharmacokinetics (DMPK) by the close of the 1990s, pharmaceutical companies are increasingly switching over to the use of statistical and knowledgebased methods, implemented in computer software, in the prediction of ADMET/DMPK properties of drug leads, in contrast to the former approach which is more costly and time consuming [14-17]. In our quest to assess the potential of natural products (NPs) derived from African medicinal plants for the development of antimalarial drugs $[18,19]$, an in silico approach based on computed molecular descriptors has been carried out, in comparison with those of known drugs, as previously described in the literature [20-24]. In this paper, we present a computer-based DMPK analysis of $>500$ anti-malarial compounds, which have been previously isolated from the African flora.

\section{Methods}

\section{Data sources}

The plant sources, geographical collection sites, chemical structures of pure compounds as well as their spectroscopic data were retrieved from literature sources comprising of MSc theses, $\mathrm{PhD}$ theses, textbook chapters and journal articles, with references ranging from 1971 to 2013. A full list of journals consulted is given in the supplementary material (Additional file 1). By convention, activities were categorized into 'very potent', 'good', 'good to moderate', 'weak', 'very weak' and 'inactive'. Following the criteria used by Mahmoudi et al. [25] and Wilcox et al. [26], a pure compound was considered highly active if $\mathrm{IC}_{50}<0.06 \mu \mathrm{M}$, being active with $0.06 \mu \mathrm{M} \leq \mathrm{IC}_{50} \leq 5 \mu \mathrm{M}$, weakly active when $5 \mu \mathrm{M} \leq$ $\mathrm{IC}_{50} \leq 10 \mu \mathrm{M}$, and compounds with IC50 > $10 \mu \mathrm{M}$ were considered inactive. The following inhibition percentages were proposed for in vivo activity of anti-malarial extracts at a fixed dose of $250 \mathrm{mg} \mathrm{kg}^{-1}$ day $^{-1}: 100 \%$ to 90\% (very good activity), $90 \%$ to $50 \%$ (good to moderate), $50 \%$ to $10 \%$ (moderate to weak) and $0 \%$ (inactive) [27].

\section{Generation of 3D models, optimization and correction of protonation states}

The 2D structures of the compounds were retrieved from the literature sources, and all 3D molecular models were generated using the graphical user interface (GUI) of the MOE software [28] running on a Linux workstation with a $3.5 \mathrm{GHz}$ Intel Core2 Duo processor. The 3D structures were generated using the builder module of MOE, and energy minimization was subsequently carried out using the MMFF94 force field [29] until a gradient of $0.01 \mathrm{kcal} \mathrm{mol}^{-1}$ was reached. The 3D structures of the compounds were then saved as .mol2 files subsequently treated with LigPrep [30]. This implementation was carried out with the GUI of the Maestro software package [31], using the optimized potentials for liquid simulations (OPLS) forcefield [32-34]. Protonation states at biologically relevant $\mathrm{pH}$ were correctly assigned (group I metals in simple salts were disconnected, strong acids were deprotonated, strong bases protonated, while topological duplicates and explicit hydrogens were added). The generated 3D models have been included in the supplementary material (Additional file 2).

\section{Calculation of molecular descriptors}

A set of ADMET-related properties (a total of 46 molecular descriptors) were calculated by using the QikProp program [35] running in normal mode. QikProp generates physically relevant descriptors and uses them to perform ADMET predictions. An overall ADMEcompliance score - drug-likeness parameter (indicated by \#stars) - was used to assess the pharmacokinetic profiles of the compounds. The \#stars parameter indicates the number of property descriptors computed by QikProp that fall outside the optimum range of values for $95 \%$ of known drugs. The methods implemented were developed by Jorgensen and Duffy [36-38]. Some of the computed ADMET descriptors are shown in Additional file 3: Table S1, along with their significance in DMPK profiling and the recommended ranges for $95 \%$ of known drugs.

\section{Results and discussion}

\section{Plant families and compound types}

From this survey, 511 anti-malarial compounds were identified from 131 plant species belonging to 45 families, a majority being isolated from the Loganiaceae family (10.6\%). Significant proportions were also identified from the Guttiferae, Zingiberaceae, Rutaceae, Asteraceae, Ancistrocladaceae, Annonaceae, Clusiaceae, Meliaceae, Sapindaceae, Lamiaceae, Moraceae, Dioncophyllaceae, Cyperaceae, Asphodelaceae, Leguminosae, Periplocaceae and Bignoniaceae families, with respective percentage compound counts of $8.0 \%, 7.1 \%, 6.9 \%$, $6.4 \%, 4.3 \%, 4.0 \%, 3.6 \%, 3.6 \%, 3.6 \%, 3.5 \%, 3.5 \%, 2.8 \%$, 
$2.6 \%, 2.4 \%, 2.4 \%, 2.1 \%$ and $2.1 \%$ of identified antimalarials (Figure 1). Less than $2 \%$ of the anti-malarials were isolated from each of the remaining plant families. A majority of the compounds were terpenoids (30.7\%), followed by alkaloids, flavonoids, quinones and xanthones, respectively representing $27.7 \%, 12.9 \%, 4.5 \%$ and $4.5 \%$ by compound count (Figure 2). Only 20 compounds were identified with in vivo anti-malarial activities, while 278 compounds showed in vitro activities from moderate to very high activities. These have been discussed in our previous review articles in this series $[18,19]$.

Significance of selected computed molecular descriptors Selected molecular descriptors, along with the recommended range of values for $95 \%$ of known drugs, as computed by the QikProp program [35], have been shown in Additional file 3: Table S1. According to Lipinski's 'Rule of Five', a compound is likely to be orally available when the molecular weight $(\mathrm{MW})<500$, the logarithm of the octan-1-ol/water partition coefficient $(\log \mathrm{P})<5$, the number of hydrogen donors $(\mathrm{HBD}) \leq 5$ and the number of hydrogen bond acceptors (HBA) $\leq 10$ [39]. Compounds which comply with this rule are considered to be drug-like. Moreover, Jorgensen's 'Rule of Three' has often been used as a criterion to predict oral availability of drugs [40]. According to this rule, a compound will more likely be orally available when all or some of the rules, the logarithm of the predicted aqueous solubility $\left(\log S_{\text {wat }}\right)>-5.7$, the predicted Caco-2 permeability $\left(\right.$ BIP $\left._{\text {Caco }-2}\right)>22 \mathrm{~nm} \mathrm{~s}^{-1}$ and the number of primary metabolites $<7$, are respected. The solubility calculation procedure implemented depends on the similarity property space between the given molecule and its most similar analogue within the experimental training set used to develop the model implemented in QikProp; i.e., if the similarity is $<0.9$, then the QikProp predicted value is taken, otherwise, the predicted property, $P_{\text {pred }}$, is adjusted such that

$$
P_{\text {pred }}=S P_{\exp }+(1-S) P_{\mathrm{QP}}
$$

where $S$ is the similarity, and $P_{\exp }$ and $P_{\mathrm{QP}}$ are the respective experimental and QikProp predictions for the most similar molecule within the training set. In Equation 1, if $S=1$, then the predicted property is equal to the measured experimental property of the training set compound. The predicted apparent Caco- 2 cell permeability, $\mathrm{BIP}_{\text {Caco }-2}$ (in $\mathrm{nm} \mathrm{s}{ }^{-1}$ ), models the permeability of the gut-blood barrier (for non-active transport), even though this parameter is not often correctly predicted computationally [41]. Moreover, a molecule's size, its capacity to make hydrogen bonds, its overall lipophilicity, and its shape and flexibility are important properties to consider when determining permeability. Molecular flexibility has been seen as a parameter which is dependent on the number of rotatable bonds (NRB), a property which influences bioavailability in rats [42].

The overall drug-likeness of a molecule is often determined by the \#stars parameter, which depends on 24 computed parameters (descriptors) of a molecule with respect to the recommended range for 95\% of known drugs (Additional file 3: Table S1). A \#star $=0$ corresponds to an ideally drug-like molecule, while \#stars $=n$ indicates that a given molecule has $n$ non-compliant descriptors (values fall outside the recommended range for $95 \%$ of known drugs). The solubility of a drug, evaluated by the model of Jorgensen and Duffy [36,37], determines

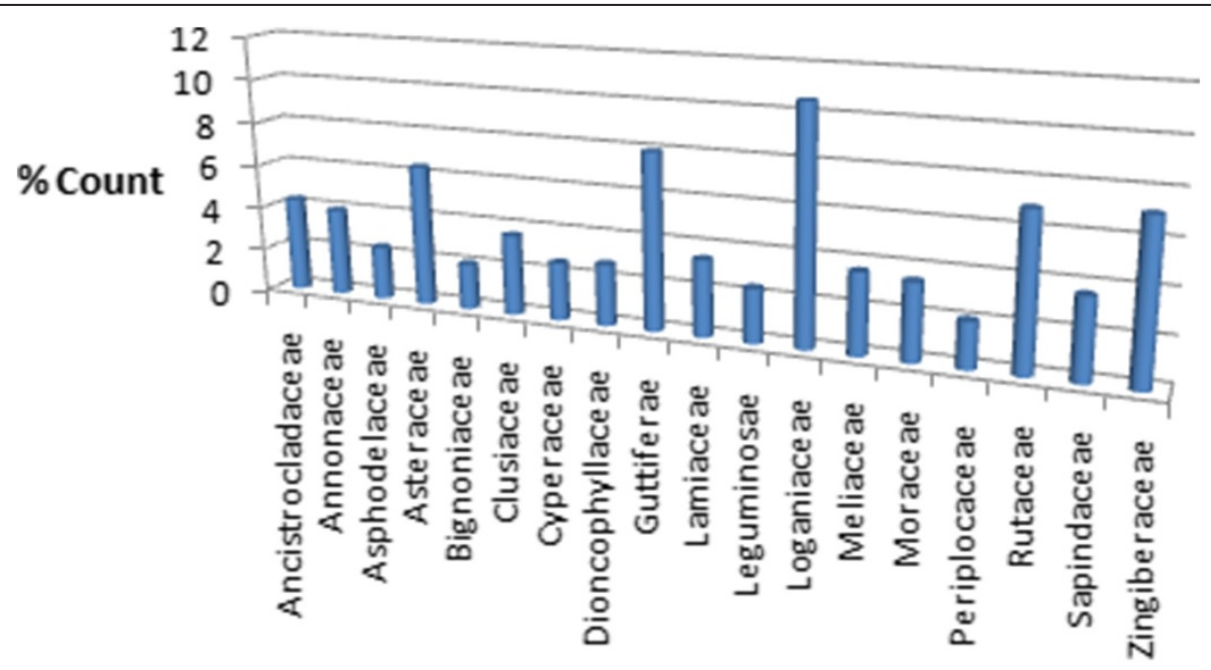

Plant families

Figure 1 Bar chart showing the distribution of the 511 anti-malarial compounds by plant family of origin. 


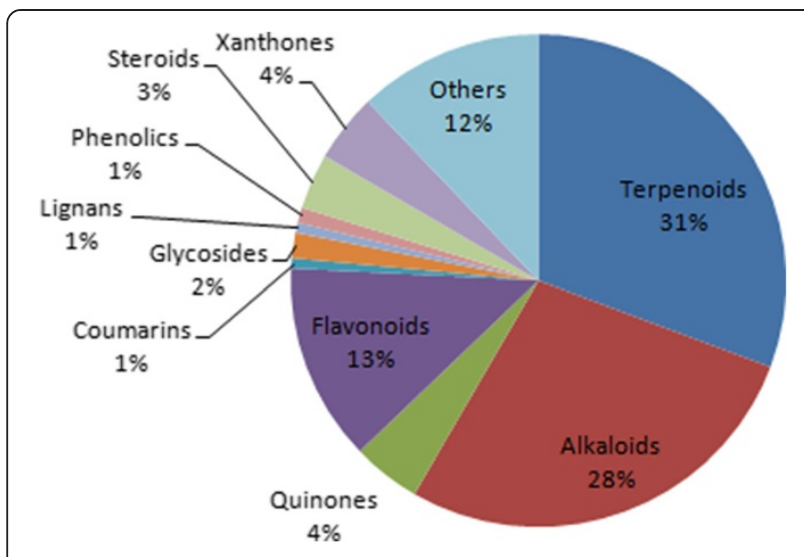

Figure 2 Pie chart showing the classification by compound types.

the bioavailability of a drug to an extent, since the bioavailability of a compound depends on the processes of absorption and liver first-pass metabolism [43]. Absorption in turn depends on the solubility and permeability of the compound, as well as interactions with transporters and metabolizing enzymes in the gut wall.

The computed blood/brain partition coefficients (log $B / B$ ) of drug molecules are often used as indicators to predict access to the central nervous system (CNS). This is because too polar drugs do not cross the blood/brain barrier (BBB). In addition, Madin-Darby canine kidney (MDCK) monolayers are widely used to make oral absorption estimates, the reason being that these cells also express transporter proteins but only express very low levels of metabolizing enzymes [42]. They are also used as an additional criterion to predict BBB penetration. Thus, our calculated apparent MDCK cell permeability could be considered to be a good mimic for the BBB (for non-active transport). The predicted skin permeability parameter $\left(\log K_{\mathrm{p}}\right)$ is another important parameter for drug distribution $[44,45]$. The predicted maximum transdermal transport rates, $J_{\mathrm{m}}$ (in $\mu \mathrm{cm}^{-2} \mathrm{~h}^{-1}$ ), were computed from the aqueous solubility $\left(\mathrm{S}_{\mathrm{wat}}\right)$ and skin permeability $\left(K_{\mathrm{p}}\right)$, using the relation (2), also expresses the efficiency of drug distribution:

$$
J_{\mathrm{m}}=K_{\mathrm{p}} \times \mathrm{MW} \times \mathrm{S}_{\mathrm{wat}}
$$

The efficiency and distribution of a drug may be affected by the degree to which it binds to the proteins within blood plasma. When a drug binds to plasma proteins (like human serum albumin, lipoprotein, glycoprotein, $\alpha, \beta$, and $\gamma$ globulins), the quantity of the drug in general blood circulation is greatly reduced and hence the less bound a drug is, the more efficiently it can traverse cell membranes. The predicted plasma-protein binding has been estimated by the prediction of binding to human serum albumin; the $\log K_{\mathrm{HSA}}$ parameter (recommended range is -1.5 to 1.5 for $95 \%$ of known drugs). The number of metabolic steps (\#metab) also determines whether the molecules can easily gain access to the target site after entering the blood stream.

The toxicity parameter is often predicted by the logarithm of $\mathrm{IC}_{50}$ values for blockage of the human ether-ago-go related gene (HERG). HERG encodes a potassium ion $\left(\mathrm{K}^{+}\right)$channel that is implicated in the fatal arrhythmia known as torsade de pointes or the long QT syndrome [46]. The HERG $\mathrm{K}^{+}$channel is best known for its contribution to the electrical activity of the heart that coordinates the heart's beating. It therefore appears to be the molecular target responsible for the cardiac toxicity of a wide range of therapeutic drugs [47]. HERG has also been associated with modulating the functions of some cells of the nervous system and with establishing and maintaining cancer-like features in leukemic cells [48]. Thus, HERG $\mathrm{K}^{+}$channel blockers are potentially toxic and the predicted $\mathrm{IC}_{50}$ values often provide reasonable predictions for cardiac toxicity of drugs in the early stages of drug discovery [49].

\section{Lipinski's criteria for evaluation of oral bioavailability}

Lipinski's 'rule' was extracted from chemical libraries from the World Dug Index (WDI) as a criterion to evaluate likely oral bioavailability $[39,50]$. This rule did not take NPs into consideration initially, since Lipinski had postulated that the Rule of Five was not respected by NPs. However, NP libraries have been previously analysed comparatively using this rule in order to have a rough idea of the extent of 'drug-likeness' of a compound library to be used in virtual screening [20-22,51,52]. In summary, 352 of the 511 compounds analysed showed no violations of Lipinski rules, while 435 compounds showed $<2$ violations. The maximum number of violations was 3 and the average number was 0.5 (Table 1). This implies that the anti-malarial compounds from the African flora could generally be categorized as 'druglike' up to approximately $70 \%$. Considering the individual parameters, it was observed that for the MW, 432 out of the 511 compounds analysed complied with

\begin{tabular}{|c|c|c|c|}
\hline Lipinski parameter & Minimum & Mean & Maximum \\
\hline $\mathrm{MW}(\mathrm{Da})$ & 78.1 & 394.1 & 1084.7 \\
\hline $\log P$ & -5.1 & 3.2 & 16 \\
\hline HBA & 0 & 6.4 & 28 \\
\hline $\mathrm{HBD}$ & 0 & 1.8 & 17 \\
\hline NRB & 0 & 5.9 & 32 \\
\hline LV & 0 & 0.5 & 3 \\
\hline
\end{tabular}

LV, Lipinski violations. 

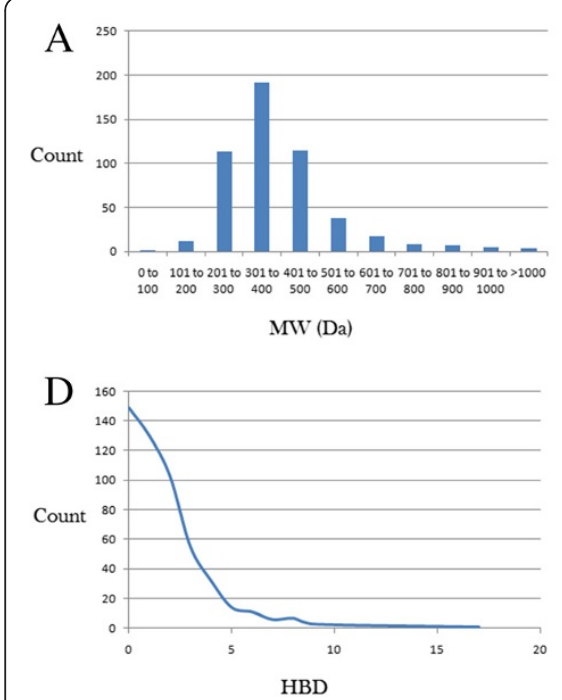

$\mathrm{B}$

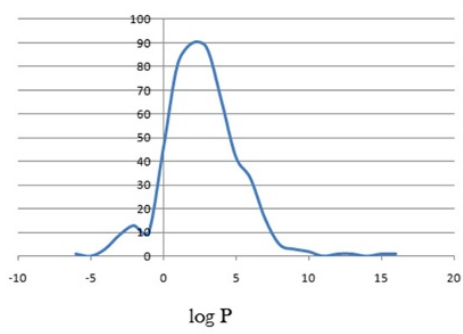

E

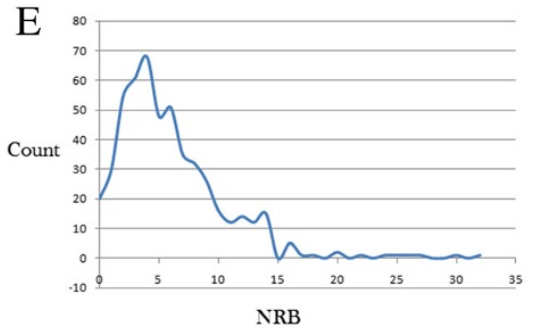

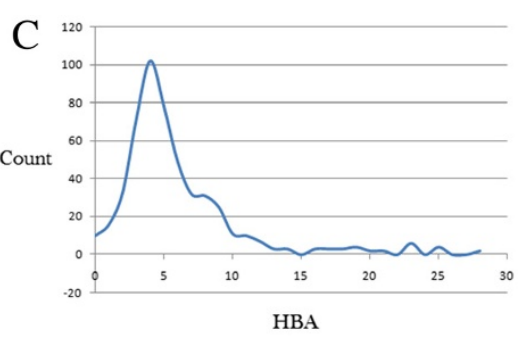

F

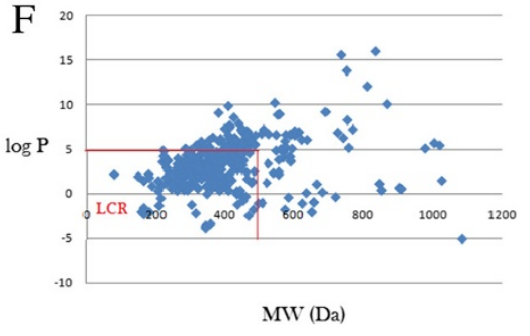

Figure 3 Graph distribution of Lipinski determinant of 'drug-likeness'. (A) Bar chart showing distribution of MW. (B, C, D and E) Distribution curves of the $\log$ P, HBA, HBD and NRB respectively for the 511 anti-malarial compounds from the African flora. (F) Scatter plot of MW against $\log$ P. For (B), the $x$-axis label is the lower limit of binned data, e.g., 0 is equivalent to 0.00 to 0.99 .

Lipinski's criterion (MW $<500 \mathrm{Da})$, constituting 84.5\%. Meanwhile, the greatest proportion of the molecules had $M W$ in the interval $301 \leq M W \leq 400$, Figure 3A. The $\log \mathrm{P}$ curve showed a Gaussian-shape, centred on a peak value of $1.5 \log \mathrm{P}$ units, with approximately $88 \%$ of the compounds complying to Lipinski's criterion $(\log \mathrm{P}<5)$, Figure 3B. The graphs of the HBA, HBD and NRB have been shown in Figure 3C,D,E. The number of HBA rose rapidly to a peak value of 4 acceptors, comprising 102 compounds, then fell off rapidly to 28 acceptors for the most complex NPs in this study. The curve for the HBD had a maximum at 0 donors (for 149 of the compounds) and fell off to 1 compound having as many as 17 donors. The distribution for the NRB gave a rugged curve with a peak value at 4 rotatable bonds (RBs), comprising 68 compounds and as many as $32 \mathrm{RBs}$ for the most flexible compound. A scatter plot of MW against log P showed that the region of maximum density of points lay in the interval $\mathrm{MW} \leq 500 \cap \log \mathrm{P} \leq 5$, defined as Lipinski's compliance region (LCR). A similar trend was seen in the pairwise scatter plots of the other parameters (results not shown).

\section{Jorgensen's criteria for assessment of oral bioavailability}

In the assessment of oral bioavailability, 279 compounds showed no violation of the Rule of Three, while 422 compounds violated this rule only once, indicating that $>80 \%$ of the compounds are predicted to be orally bioavailable. Regarding the individual parameters, the solubility parameter showed a significant performance, with about $80 \%$ of the compounds complying with the recommended range for $95 \%$ of known drugs $(-6 \leq \log$ $\left.S_{\text {wat }} \leq 0.5\right)$. In accordance to Jorgensen's ro3, $77.3 \%$ of the compounds had $\log S_{\text {wat }}>5.7$, while $91 \%$ complied with the BIP $_{\text {Caco - 2 }}>22 \mathrm{~nm} \mathrm{~s}^{-1}$ criterion. The percentage of compounds complying with each of the other DMPK parameters has been shown in Table 2. The distribution curves of both parameters are shown in Figure 4A,B, along with the histogram for the human oral absorption (HOA) parameter. It was estimated that 312 compounds

Table 2 Mean values and percentage compliances of selected ADMET-related descriptors for 511 anti-malarial compounds from African medicinal plants

\begin{tabular}{lll}
\hline Property $^{\mathbf{a}}$ & Mean value & Percentage compliance \\
\hline $\log B / B$ & -1.0 & 93.3 \\
BIP $_{\text {Caco-2 }}\left(\mathrm{nm} \mathrm{s}^{-1}\right)$ & $1,139.0$ & 14.1 \\
$S_{\text {mol }}\left(\AA^{2}\right)$ & 641.5 & 95.1 \\
$S_{\text {mol, hfob }}\left(\AA^{2}\right)$ & 350.0 & 95.5 \\
$V_{\text {mol }}\left(\AA^{3}\right)$ & $1,202.3$ & 94.5 \\
$\log S_{\text {wat }}\left(S\right.$ in mol L $\left.{ }^{-1}\right)$ & -4.3 & 79.3 \\
$\log K_{\text {HSA }}$ & 0.3 & 83.6 \\
MDCK & 627.8 & 54.0 \\
Ind & 0.012 & 95.3 \\
Glob & 0.9 & 92.8 \\
QP & 39.6 & 94.1 \\
logHERG & -4.6 & 57.3 \\
$\log K_{\mathrm{p}}$ & -3.2 & 93.3 \\
\#metab & 5.4 & 84.5 \\
\hline
\end{tabular}

The descriptors (entries in the first column and the recommended ranges for 95\% of drugs) are defined in Additional file 3: Table S1. 

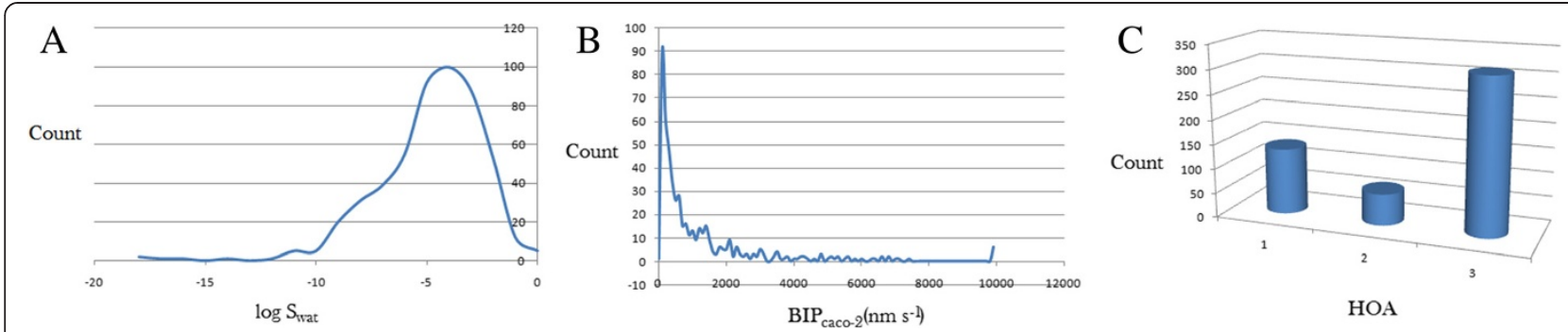

Figure 4 Distribution of parameters for oral bioavailability and human oral absorption. (A) $\log S_{\text {wat }}$ (B) BIP Caco-2 and (C) HOA. For the HOA parameter (C), 1, low absorption; 2, medium absorption; 3, high absorption.

could have high HOA (attributed to a value of 3 on Figure $4 \mathrm{C}$ ), while 64 compounds were estimated to have medium HOA (attributed to a value of 2) and 135 compounds were estimated to have low absorption. Estimations of the percentage of human oral absorption (PHOA) showed that $38.1 \%$ of the compounds could be absorbed at $100 \%$, while $52.2 \%$ could be absorbed at $>90 \%$ and $69.9 \%$ were estimated to have high PHOA (i.e., absorbed at $>80 \%$ ).

\section{Assessment of the overall DMPK compliance by the drug-likeness parameter}

The \#stars parameter was also used to evaluate the overall drug-likeness of the anti-malarial compounds from African flora. The distribution curve for this parameter has been shown in Figure 5. The graph started with 0 violations of drug-likeness indicators (corresponding to a maximum number of 282 compounds) and went up to 15 violations. The steepness of the curve, however, showed that the compounds had an overall interesting drug-likeness. Additionally, $79.2 \%$ of the compounds violated only $\leq 2$ out of the recommended range of values for the 24 essential descriptors of drug-likeness computed by QikProp in this study.
Prediction of drug distribution and binding to human serum albumin and dermal penetration parameters

The distribution of the compounds was simulated by calculation of binding affinities to human serum albumin (HSA). The distribution of log $K_{\mathrm{HSA}}$ gave a smooth Gaussian-shaped curve with a peak value at $0.5 \log K_{\mathrm{HSA}}$ units (Figure 6A), with $83.6 \%$ of the compounds falling within the required range for $95 \%$ of drugs. This is an indication that a significant proportion of the compounds are likely to circulate freely in the blood stream and hence reach the drug target sites.

The skin permeability parameter showed $93.3 \%$ compliance with $95 \%$ of drugs. The distribution curve was a Gaussian-shape centred on $-2.5 \log K_{\mathrm{p}}$ units (Additional file 3: Figure $\mathrm{S} 1$ ), while the predicted maximum transdermal transport rates, $J_{\mathrm{m}}$ (in $\mu \mathrm{cm}^{-2} \mathrm{~h}^{-1}$ ), were estimated to vary from 0 to about 1,640 units, with only five compounds having predicted value of $J_{\mathrm{m}}>100 \mu \mathrm{cm}^{-2} \mathrm{~h}^{-1}$.

\section{Prediction of blood/brain barrier penetration and activity} in the central nervous system

Access to the central nervous system was simulated by the $\log B / B$ parameter, while activity in the central nervous system was estimated by the CNS parameter (on the $-2=$

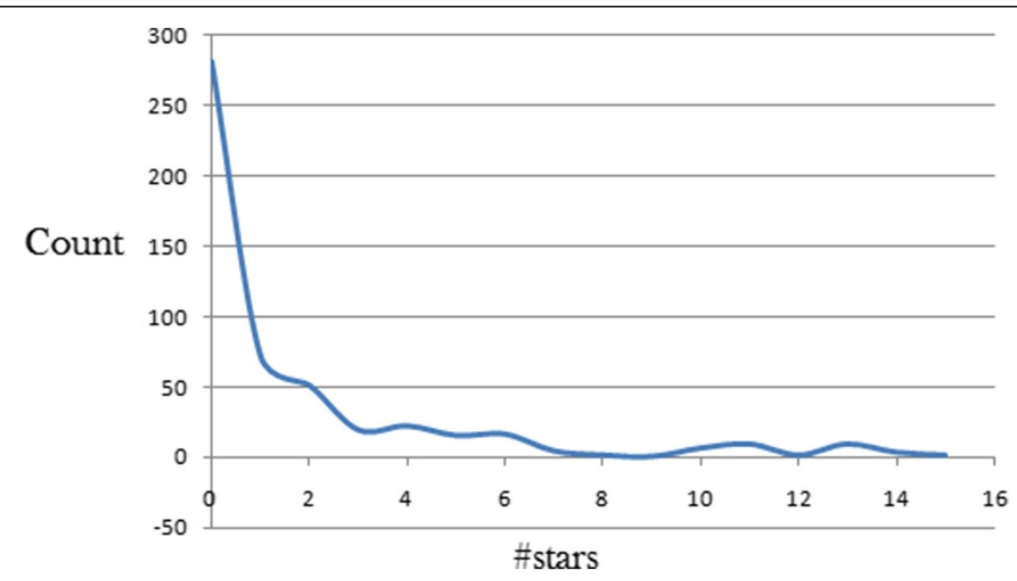

Figure 5 Distribution curves for \#stars for 511 anti-malarial compounds derived from African medicinal plants. 

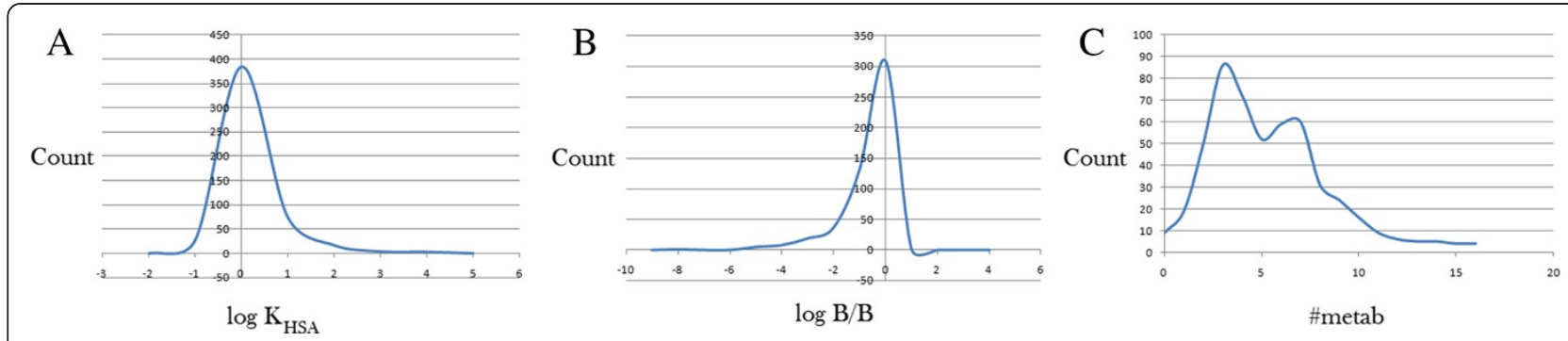

Figure 6 Plot of the physicochemical descriptors used to predict DMPK. (A) Predicted log $K_{\text {HSA }}$ against count, (B) Predicted log B/B against count and (C) Predicted \# metab against count.

inactive and $+2=$ active scale). The $\log B / B$ plot (Figure $6 \mathrm{~B}$ ) showed a sharp peak at $0.5 \log B / B$ units, with $93.3 \%$ compliance with $95 \%$ of drugs. On the other hand, only $3.5 \%$ of the compounds were estimated to have an activity in the central nervous system.

\section{Prediction of number of likely metabolic reactions}

The number of likely metabolic steps was also computed by QikProp and plotted against the counts (Figure 6C). This parameter is often used to assess the likelihood of a molecule to easily gain access to the target site after entering the blood stream. The average estimated number of possible metabolic reactions was between 5 and 6 , even though some of the compounds are likely to undergo as many as up to 16 metabolic reactions due to the complexity of some of the plant secondary metabolites. About $84 \%$ of the compounds are predicted to undergo the recommended number of metabolic steps (1 to 8 reactions),

\section{Prediction of toxicity by blockage of HERG $\mathrm{K}^{+}$channel}

In this study, the estimated or predicted $\mathrm{IC}_{50}$ values for blockage of the HERG $\mathrm{K}^{+}$channel have been used to model the drug toxicity in silico. The recommended range for predicted $\log \mathrm{IC}_{50}$ values for blockage of HERG $\mathrm{K}^{+}$channels (logHERG) is $>-5$. A distribution curve for the variation of the predicted logHERG is shown in Figure 7, which is a left-slanted Gaussianshaped curve, with a peak at $-5.5 \operatorname{logHERG}$ units. It was observed that in general, this parameter is predicted to fall within the recommended range for about $57 \%$ of the compounds.

\section{Conclusions}

In this study, the calculated physicochemical properties and indicators of drug-likeness have been used in the assessment of the ADMET/DMPK profiles of $>500$ compounds isolated from medicinal plants in Africa, which have exhibited from weak to high in vitro and/or in vivo anti-plasmodial/anti-malarial activity. The overall estimate gave good compliance with the computed parameters for $95 \%$ of known drugs, as well as with Lipinski' drug-likeness criteria. The results give good reason for further investigation of the suitability of anti-malarial compounds derived from African flora to be directly employed as drugs or as lead compounds from which

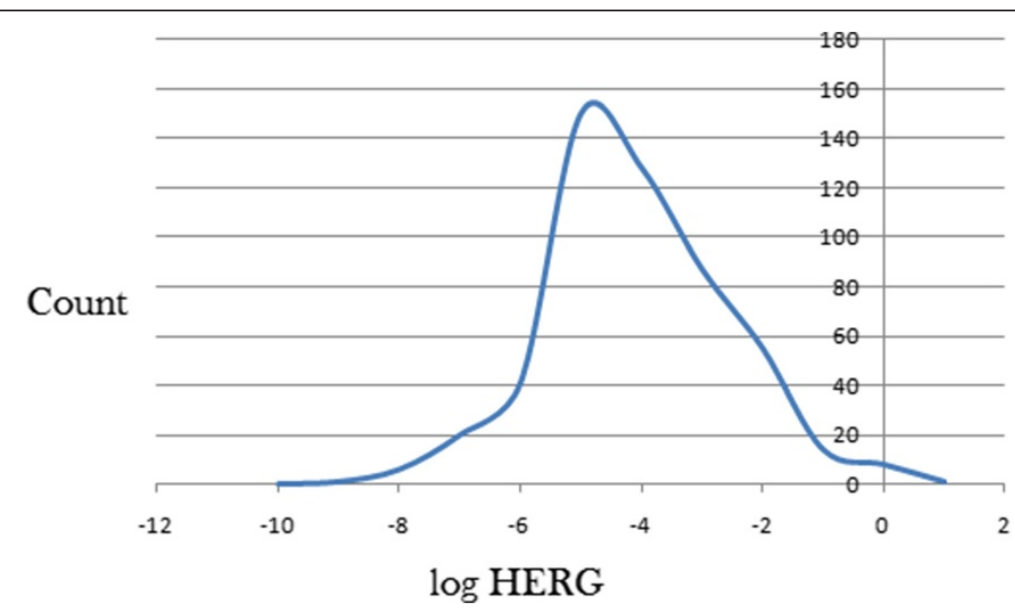

Figure 7 A plot of predicted logHERG values for 511 anti-malarial compounds derived from the African flora. 
new anti-malarial drugs could be developed. The generated 3D structures of the compounds have been included as a supplementary file (Additional file 2), while enquiries for the availability of compounds for screening purposes could be addressed to the p-ANAPL consortium [52], which has the mandate to collect compound samples from African flora for biological screening purposes.

\section{Additional files}

Additional file 1: List of journals consulted in the literature search.

Additional file 2: The 3D structures of the anti-malarial compounds isolated from African medicinal plants in this survey. This file is saved in .sdf format (which can be viewed using a wide range of software, including MOE, LigandScout, Discovery Studio, and Maestro).

Additional file 3: Table S1. Selected computed ADMET-related descriptors and their recommended ranges for $95 \%$ of known drugs. Figure S1. Distribution curves for the predicted skin permeability parameter.

\section{Abbreviations}

3D: three-dimensional; ADMET: absorption, distribution, metabolism, excretion and toxicity; DMPK: drug metabolism and pharmacokinetics; HBA: hydrogen bond acceptors; HBD: hydrogen bond donors; log P: logarithm of the octan-1-ol/water partition coefficient; MDCK: Madin-Darby canine kidney; MW: molecular weight; NP: natural product; NRB: number of rotatable bonds.

\section{Competing interests}

The authors declare no competing interests.

\section{Authors' contributions}

FNK, LLL, JAM, JCN and LMM conceived the idea. FNK, LLL and PAO participated in the data collection and molecular simulation experiments. FNK, JAM and PAO contributed in the data analysis, the discussion of results and the conception of the paper under the supervision of LMM, WS, LLL and $J C N$. FNK and PAO wrote the first draft of the paper, and all authors agreed on the final version before submission. This work is part of the PhD of PAO. All authors read and approved the final manuscript.

\section{Acknowledgements}

Financial support is acknowledged from Lhasa Ltd, Leeds, UK through the Chemical and Bioactivity Information Centre (CBIC), University of Buea, Cameroon. Schrodinger Inc. is acknowledged for the academic license.

\section{Author details}

${ }^{1}$ Department of Chemistry, Faculty of Science, University of Douala, 00237 , P. O. Box 24157 Douala, Cameroon. ${ }^{2}$ Department of Chemistry, Faculty of Science, Chemical and Bioactivity Information Centre, University of Buea, 00237, P. O. Box 63 Buea, Cameroon. ${ }^{3}$ Department of Pharmaceutical Sciences, Martin-Luther University of Halle-Wittenberg, Wolfgang-Langenbeck Str. 4, Halle (Saale) 06120, Germany.

Received: 14 April 2014 Accepted: 26 June 2014

Published online: 05 September 2014

\section{References}

1. Malaria atlas project (MAP). http://www.map.ox.ac.uk. Accessed 2 August 2013

2. WHO - World Health Organization (2012) World malaria report 2012. Geneva, Available from http://who.int/malaria/publications/world_malaria_ report_2012/wmr2012_no_profiles.pdf. Accessed 2 August 2013

3. Vogel $\mathrm{G}$ (2010) Infectious disease - new map illustrates risk from the 'other' malaria. Science 329:618-618
4. Hoffman SL, Subramanian GM, Collins FH, Venter JC (2002) Plasmodium, human and Anopheles genomics and malaria. Nature 415:702-709

5. White NJ (2004) Antimalarial drug resistance. J Clin Invest 113:1084-1092

6. Anthony MP, Burrows JN, Duparc S, Moehrle J, Wells TNC (2012) The global pipeline of new medicines for the control and elimination of malaria. Malar J 11:316

7. Wongsrichanalai C, Pickard AL, Wernsdorfer WH, Meshnick SR (2002) Epidemiology of drug-resistant malaria. Lancet Infect Diseases 2:209-218

8. DiMasi JA, Hansen RW, Grabowsk HG (2003) The price of innovation: new estimates of drug development costs. J Health Econ 22:151-185

9. Sullivan DJ Jr, Kaludov N, Martinov MN (2011) Discovery of potent, novel, non-toxic anti-malarial compounds via quantum modelling, virtual screening and in vitro experimental validation. Malar J 10:274

10. Singh N, Cheve G, Avery MA, McCurdy CR (2007) Targeting the methyl erythritol phosphate (MEP) pathway for novel antimalarial, antibacterial and herbicidal drug discovery: inhibition of 1-deoxy-D-xylulose-5-phosphate reductoisomerase (DXR) enzyme. Curr Pharm Des 13:1161-1177

11. Griffith $R$, Chanphen R, Leach SP, Keller PA (2002) New anti-malarial compounds from database searching. Bioorg Med Chem Lett 12(4):539-542

12. Bhattacharjee AK, Geyer JA, Woodard CL, Kathcart AK, Nichols DA, Prigge ST, Li Z, Mott BT, Waters NC (2004) A three-dimensional in silico pharmacophore model for inhibition of Plasmodium falciparum cyclin-dependent kinases and discovery of different classes of novel Pfmrk specific inhibitors. J Med Chem 47(22):5418-5426

13. Jacq N, Salzemann J, Jacq F, Legré Y, Medernach E, Montagnat J, Maaß A, Reichstadt M, Schwichtenberg H, Sridhar M, Kasam V, Zimmermann M, Hofmann M, Breton V (2008) Grid-enabled virtual screening against malaria. Journal of Grid Computing 6(1):29-43

14. Hecht D, Cheung M, Fogel GB (2008) QSAR using evolved neural networks for the inhibition of mutant PfDHFR by pyrimethamine derivatives Biosystems 92(1):10-15

15. Darvas F, Keseru G, Papp A, Dormán G, Urge L, Krajcsi P (2002) In silico and ex silico ADME approaches for drug discovery. Top Med Chem 2:1287-1304

16. Hodgson J (2001) ADMET - turning chemicals into drugs. Nat Biotechnol 19:722-726

17. Cronin MTD (2003) Computer-assisted prediction of drug toxicity and metabolism in modern methods of drug discovery. In: Hilgenfeld R, Hillisch A (eds) Modern methods of drug discovery. Birkhäuser, Basel

18. Amoa Onguéné P, Ntie-Kang F, Lifongo LL, Ndom JC, Sippl W, Mbaze LM (2013) The potential of anti-malarial compounds derived from African medicinal plants, part I: a pharmacological evaluation of alkaloids and terpenoids. Malar J 12:449

19. Ntie-Kang F, Amoa Onguéné P, Lifongo LL, Ndom JC, Sippl W, Mbaze LM (2014) The potential of anti-malarial compounds derived from African medicinal plants, part II: a pharmacological evaluation of non-alkaloids and non-terpenoids. Malar J 13:81

20. Ntie-Kang F, Mbah JA, Mbaze LM, Lifongo LL, Scharfe M, Ngo Hanna J, Cho-Ngwa F, Amoa Onguéné P, Owono LC, Megnassan E, Sippl W, Efange SMN (2013) CamMedNP: building the Cameroonian 3D structural natural products database for virtual screening. BMC Complement Altern Med 13:88

21. Ntie-Kang F, Onguéné PA, Scharfe M, Owono LCO, Megnassan E, Mbaze LM, Sippl W, Efange SMN (2014) ConMedNP: a natural product library from central African medicinal plants for drug discovery. RSC Adv 4:409-419

22. Ntie-Kang F, Zofou D, Babiaka SB, Meudom R, Scharfe M, Lifongo LL, Mbah JA, Mbaze LM, Sippl W, Efange SMN, AfroDb (2013) A select highly potent and diverse natural product library from African medicinal plants. PLOS ONE 8(10):e78085

23. Ntie-Kang F, Mbah JA, Lifongo LL, Owono LCO, Megnassan E, Mbaze LM, Judson PN, Sippl W, Efange SMN (2013) Assessing the pharmacokinetic profile of the CamMedNP natural products database: an in silico approach. Org Med Chem Lett 3:10

24. Ntie-Kang F, Lifongo LL, Mbah JA, Owono LCO, Megnassan E, Mbaze LM, Judson PN, Sippl W, Efange SMN (2013) In silico drug metabolism and pharmacokinetic profiles of natural products from medicinal plants in the Congo basin. In Silico Pharmacology 1:12

25. Mahmoudi N, de Julian-Ortiz JV, Cicerone L, Galvez J, Mazier D, Danism M, Derouin F, Garcia-Domenech R (2006) Identification of new antimalarial drugs by linear discriminant analysis and topological virtual screening. J Antimicrob Chemother 57:489-497

26. Willcox M, Bodeker G, Rasanaivo P (2004) Traditional medicinal plants and malaria. CRC, Paris 
27. Rasoanaivo P, Oketch-Rabah H (1998) Preclinical considerations on anti-malarial phytomedicines: part II, efficacy evaluation. Institut Malgache des Recherches Appliquées, Antananarivo

28. Chemical Computing Group Inc (2010) Molecular operating environment software. Chemical Computing Group Inc, Montreal

29. Halgren TA (1996) Merck molecular forcefield. J Comput Chem 17:490-641

30. Schrödinger (2011) LigPrep software, version 2.5. LLC, New York

31. Schrödinger (2011) Maestro, version 9.2. LLC, New York

32. Shivakumar D, Williams J, Wu Y, Damm W, Shelley J, Sherman W (2010) Prediction of absolute solvation free energies using molecular dynamics free energy perturbation and the OPLS force field. J Chem Theory Comput 6:1509-1519

33. Jorgensen WL, Tirado-Rives J (1988) The OPLS [optimized potentials for liquid simulations] potential functions for proteins, energy minimizations for crystals of cyclic peptides and crambin. J Am Chem Soc 110(6):1657-1666

34. Jorgensen WL, Maxwell DS, Tirado-Rives J (1996) Development and testing of the OPLS all-atom force field on conformational energetics and properties of organic liquids. J Am Chem Soc 118(45):11225-11236

35. Schrödinger (2011) QikProp, version 3.4. LLC, New York

36. Jorgensen WL, Duffy EM (2000) Prediction of drug solubility from Monte Carlo simulations. Bioorg Med Chem Lett 10:1155-1158

37. Jorgensen WL, Duffy EM (2002) Prediction of drug solubility from structure. Adv Drug Deliv Rev 54:355-366

38. Duffy EM, Jorgensen WL (2000) Prediction of properties from simulations: free energies of solvation in hexadecane, octanol, and water. J Am Chem Soc 122:2878-2888

39. Lipinski CA, Lombardo F, Dominy BW, Feeney PJ (1997) Experimental and computational approaches to estimate solubility and permeability in drug discovery and development settings. Adv Drug Delivery Rev 23:3-25

40. Schrödinger (2011) QikProp 3.4 user manual. LLC, New York

41. Tetko IV, Bruneau P, Mewes H-W, Rohrer DC, Poda Gl (2006) Can we estimate the accuracy of ADMET predictions? Drug Discov Today 11:700-707

42. Veber DF, Johnson SR, Cheng HY, Smith BR, Ward KW, Kopple KD (2002) Molecular properties that influence the oral bioavailability of drug candidates. J Med Chem 45:2615-2623

43. Van de Waterbeemd H, Gifford E (2003) ADMET in silico modelling: towards prediction paradise? Nat Rev Drug Discov 2:192-204

44. Potts RO, Guy RH (1992) Predicting skin permeability. Pharm Res 9:663-669

45. Potts RO, Guy RH (1995) A predictive algorithm for skin permeability: the effects of molecular size and hydrogen bond activity. Pharm Res 12:1628-1633

46. Hedley PL, Jørgensen P, Schlamowitz S, Wangari R, Moolman-Smook J, Brink PA, Kanters JK, Corfield VA, Christiansen M (2009) The genetic basis of long QT and short QT syndromes: a mutation update. Hum Mutat 30:1486-1511

47. Vandenberg Jl, Walker BD, Campbell TJ (2001) HERG $\mathrm{K}^{+}$channels: friend or foe. Trends Pharmacol Sci 22:240-246

48. Chiesa N, Rosati B, Arcangeli A, Olivotto M, Wanke E (1997) A novel role for HERG K+ channels: spike-frequency adaptation. J Physiol 501:313-318

49. Aronov AM (2005) Predictive in silico modeling for hERG channel blockers. Drug Discov Today 10:149-155

50. Lipinski CA (2000) Drug-like properties and the causes of poor solubility and poor permeability. J Pharmacol Toxicol Methods 44:235-249

51. Quinn RJ, Carroll AR, Pham MB, Baron P, Palframan ME, Suraweera L, Pierens GK, Muresan S (2008) Developing a drug-like natural product library. J Nat Prod 71:464-468

52. Ntie-Kang F, Amoa Onguéné $P$, Fotso GW, Andrae-Marobela K, Bezabih M, Ndom JC, Ngadjui BT, Ogundaini AO, Abegaz BM, Mbaze LM (2014) Virtualizing the p-ANAPL library: a step towards drug discovery from African medicinal plants. PLoS ONE 9:e90655

doi:10.1186/s13588-014-0006-x

Cite this article as: Onguéné et al:: The potential of anti-malarial compounds derived from African medicinal plants, part III: an in silico evaluation of drug metabolism and pharmacokinetics profiling. Organic and Medicinal Chemistry Letters 2014 4:6.

\section{Submit your manuscript to a SpringerOpen ${ }^{\odot}$ journal and benefit from:}

- Convenient online submission

- Rigorous peer review

- Immediate publication on acceptance

- Open access: articles freely available online

- High visibility within the field

- Retaining the copyright to your article

Submit your next manuscript at $\gg$ springeropen.com 